\title{
Afecção do tendão supra-espinal e afastamento laboral
}

\author{
Supraspinatus tendon affection and sick leave
}

\author{
Josiane Schadeck de Almeida ${ }^{1}$ \\ Guaracy Carvalho Filho ${ }^{1}$ \\ Carlos M arcelo Pastre ${ }^{2}$ \\ Neuseli M arino Lamari $^{3}$ \\ Eliane Cristina Pastre ${ }^{1}$
}

${ }^{1}$ Departamento de

OrtopediaeTraumatologia, FAM ERP. Av. Brigadeiro Faria Lima 5416,

Universitário. 15090-000 São José do Rio Preto SP. schaj24@hotmail.com

${ }^{2}$ Departamento de Fisioterapia, Faculdade de Ciências eTecnologia/ UNESP.

${ }^{3}$ Departamento dePósGraduação. Faculdadede M edicina de São J osé do Rio Preto.
Abstract Rotator cuff disease, among others damage of the supraspinatus tendon mainly caused by work overload, is a common problem in the population resulting in a high incidence of sick leaves. In the present survey we sought to compare the need for sick leaves in relation to different stages of supraspinatus tendon affection and in relation to five different groups of workers. Our study counted with the participation of patients who were diagnosed with this condition. The individuals were grouped according to stages of the disease (tendonitis, partial rupture, total rupture) and according to the biomechanical aspects of their occupation (general services, civil construction, domestic workers, farm workers and security guard services). Statistical analysis was performed using Pearson'schi-squaretest, dependence analysis and exact test. Results revealed that 62 (55\%) of the individuals were on sick leave. The highest rates of sick leaves according to Pearson's test occurred in the groups general services $(38,71 \%)$ and farm workers (22,58\%). According to the dependence analysis, tendonitis $(p<0,05)$ was the most frequent reason for sick leaves and, according to Pearson's test, farm work seems to keep the individual for a longer space of time unfit for duty $(p=0,02)$.

Key words Shoulder pain, Shoulder impingement syndrome, Sick leave
Resumo As afecções do manguito rotador, dentre elas as relacionadas ao tendão supra-espinal, são problemas comuns na população, sobretudo devido à sobrecarga ocupacional, o que leva a altos índices de afastamento do trabalho. Buscouse, então, comparar a necessidade deafastamento de trabalho entre os diferentes estados da afecção do tendão supra-espinal e entre cinco diferentes grupos profissionais, tendo a participação de pacientes que apresentavam diagnóstico da afecção. O sindivíduos foram agrupados quanto ao estado da doença (tendinite, ruptura parcial, ruptura total) e quanto aos aspectos biomecânicos da ocupação (ramo de serviços, construção civil, trabaIhadores domésticos, lavradores e seguranças). Testequi-quadrado dePearson, análise de dependência e teste exato para uma proporção foram realizados. Osresultadosapontaram que62 (55\%) estavam afastados da atividade laboral e que os grupos com maior número de afastados foram 0 do ramo de serviços $(38,71 \%)$ e lavradores $(22,58 \%)$, segundo Pearson. A maior freqüência de casos de afastamento foi registrada no estágio de tendinite $(p<0,05)$ pela análise de dependência ea ocupação delavrador parece deixar o indivíduo por mais tempo afastado $(p=0,02)$, segundo teste de Pearson.

Palavras-chave Dor de ombro, Síndrome de colisão do ombro, Licença médica 
Introdução

As lesões do manguito rotador são problemas comuns relacionados a sobrecargas na articulação do ombro, sobretudo em condições ocupacionais ${ }^{1}$. Esse agravo é responsável por considerável número de afastamentos do trabalho, cerca de $27 \%$, resultando em custos ao sistema previdenciário e, também, problemas sociais, como dificuldade de reinserção no mercado formal de emprego ${ }^{2-6}$.

Estas lesões representam um espectro de doenças quevão desdeuma tendinite, passando por uma ruptura parcial até uma lesão de ruptura total comprometendo todos dos componentes do manguito rotador?.

Várias etiopatogenias estão relacionadas com os distúrbios osteomusculares relacionados ao trabalho (DORT) do ombro, como posturas inadequadas dos membros superiores, tipo de ocupação ${ }^{8,9}$, repetitividade de movimentos acima do nível do ombro ${ }^{10-12}$ eenvel hecimento biológico ${ }^{13,14}$.

Sua característica multifatorial é fator de complicação para o entendimento egerenciamento das lesões, principalmente considerando a diversidade cinesi ológica envolvida nas mais variadas formas de ocupação laboral 15-17.

Baseando-se nas questões anteriormente amealhadas que definem as doenças em questão como situação problema relacionada a atividades ocupacionais eque descrevem a variedade de manifestações evolutivas e condições causais do agravo como fator de complicação para o gerenciamento de medidas preventivas e de reabilitação, entende-se como pertinente investigar os aspectos relacionados à doença e sua repercussão sobre o trabalho.

Assim, constituem-se como os objetivos deste estudo a comparação da necessidade de afastamento de trabalho entre os diferentes estágios evolutivos da doença eentre cinco diferentes grupos profissionais com diferentes aspectos biomecânicos da ocupação.

\section{M étodos}

Foram convidados a participar do estudo todos os indivíduos atendidos no Hospital de Base da Faculdade de M edicina de São J osé do Rio Preto com o diagnóstico de afecção do manguito rotador durante os meses de abril e novembro de 2004, que foram informados sobre o objetivo da pesquisa. A pós a assinatura do termo de consentimento livre e esclarecido, foi feita a entrevista seguindo um roteiro de entrevista previamente elaborado.

Estes pacientes possuíam história de dor espontânea, arco doloroso na elevação de membro superior e positividades no testeirritativo de $\mathrm{Neer}^{1}$.

Para a participação, os pacientes passaram pela avaliação clínica do ortopedista do local do estudo, onde também foi realizado o exame de ultra-som e/ou de ressonância magnética de ombro para confirmação do diagnóstico. Os achados alterados ou positivos foram interpretados como tendinite do supra-espinal, ruptura parcial ou total do supra-espinal ${ }^{15}$. Foram considerados critérios de exclusão a história de cirurgias ou fraturas anteriores no ombro, instabilidades articulares e afecção em mais de um tendão, já que o objetivo do estudo visa à estrutura particular do tendão supra-espinhal.

A abordagem foi feita durantea consulta promovida pelos ortopedistas do departamento. Os dados utilizados foram coletados por meio de entrevista com questionário previamente elaborado. 0 instrumento continha questões referentes aos aspectos pessoais (idade, sexo, peso, membro superior dominante, lado da afecção) atividadeocupacional (função no trabalho, tempo de serviço, função anterior, acúmulo de função) e afastamento laboral (tempo deafastamento), feito apenas por um pesquisador. As informações sobre o estágio da lesão, registradas em prontuários clínicos, foram anotadas na ficha da entrevista.

Os participantes foram agrupados conforme o estado da patologia do tendão supra-espinhal (tendinite, ruptura parcial eruptura total) ${ }^{15}$ edeacordo com a atividadelaboral exercida, com ênfase na biomecânica ${ }^{14}$, constituindo-se de cinco grupos: a) indivíduos do ramo de serviços ( costureira, cabeleireiro e do lar); b) construção civil (pedreiro, marceneiro e serralheiro); c) trabalhadores domésticos e faxineiros; d) lavradores; e) seguranças.

O estudo teve aprovação pela Comissão de Ética em Pesquisa da Faculdade de Medicina de São José do Rio Preto, com protocolo no 1941/ 2004 de 12 de abril. A abordagem dos achados como idade e grupamento ocupacional foi realizada por meio da média, mediana e desvio padrão. Entre as variáveis afastamento laboral e estado da doença, utilizou-se a análise de dependência ${ }^{18}$; 0 teste exato para uma proporção foi usado na variável lateralidade da queixa e membro dominante. Realizou-se a caracterização por grupamento ocupacional, afastamento do trabalho e tempo de afastamento pelo teste qui- 
quadrado de Pearson. 0 nível de significância adotado foi $p<0,05$.

\section{Resultados}

Do total de participantes, excluíram-se doze indivíduos que não preenchiam os critérios de inclusão, permanecendo 112, sendo 75 (67\%) do sexo feminino e $37(33 \%)$ do masculino, cuja idade variou de 23 a 71 anos, com média de 51 anos (desvio padrão de 9 anos). D esta população, $96 \%$ (107) eram destros e o membro acometido direito foi observado em $69 \%$ (78), sendo maisfreqüente pelo teste exato para uma proporção $(p<0,05)$.

Em relação à atividade laboral, a amostra foi dividida de acordo com o ramo de atividade e com o tipo de movimento biomecânico exercido. O grupo I foi formado por 58 indivíduos (52\%) que exercem ou exerceram trabalhos manuais como costureira, cabeleireiro e indivíduos que trabalham no lar. O grupo II constituiu-se de quinze indivíduos (13\%) queexercem ou exerceram a função de pedreiro, marceneiro e serraIheiro. 0 grupo III constituiu-sededezenovepes- soas que trabalham como faxineiro e trabalhador doméstico (17\%). 0 grupo IV foi formado por dezoito lavradores (16\%) e o grupo $\mathrm{V}$ por dois seguranças (2\%). Dentre todos, 62 (55\%) afastaram-se da atividade laboral.

A Tabela 1 apresenta a distribuição dos participantes quanto à ocupação e idade, agrupada conforme aspectos biomecânicos. N ota-se que a faixa de média de idades independente da população encontra-se entre 45 e 53 anos enão houve diferenças estatisticamente significativas entre idade e ocupação.

Em relação ao afastamento laboral, observasena Tabela 2 quea maior freqüência de casos de afastamento foi registrada no estado de tendinite $(p<0,05)$, segundo análisede dependência. Para os outros estados, as distribuições foram casuais, sem diferença estatística.

$\mathrm{Na}$ Tabela 3, éapresentada a distribuição dos participantes quanto à sua atividade ocupacional e necessidade de se afastar do trabalho. Observa-se valor significante para os grupos I e IV, mostrando maior afastamento laboral $(p=0,024)$, segundo teste de Pearson.

A Tabela 4 apresenta a distribuição dos indivíduos afastados que tinham mais de 49 meses

Tabela 1. Distribuição de freqüência da variável grupo de ocupação e respectivas médias de idade e desviospadrão para cada grupo.

\begin{tabular}{lcrc}
\hline \multicolumn{1}{c}{ Ocupação } & Grupamento profissional & Idade & Total de indivíduos \\
\hline Costureira, cabeleireiro e do lar & Grupo I & $52 \pm 10$ & $58(52 \%)$ \\
Construção civil & Grupo II & $45 \pm 9$ & $15(13 \%)$ \\
Trabalhador doméstico & Grupo III & $53 \pm 8$ & $19(17 \%)$ \\
Lavrador & Grupo IV & $53 \pm 11$ & $18(16 \%)$ \\
Segurança & Grupo V & $50,5 \pm 0,7$ & $02(2 \%)$
\end{tabular}

Nota: A taxa de indivíduos por grupo foi formada com relação ao aspecto biomecânico, não encontrando diferença de idade.

Tabela 2. Distribuição das lesões segundo estado da doença e necessidade de afastamento.

\begin{tabular}{|c|c|c|c|}
\hline \multirow{2}{*}{ Estado da afecção } & \multicolumn{2}{|c|}{ Necessidade de afastamento } & \multirow{2}{*}{ Total de lesões } \\
\hline & Sim & Não & \\
\hline Tendinite & $48,39 \%(30)^{*}$ & $32,0 \%(16)$ & $41,70 \%(46)$ \\
\hline Ruptura parcial & $32,26 \%(20)$ & $60,0 \%(30)$ & $44,64 \%(50)$ \\
\hline Ruptura total & $19,35 \%(12)$ & $8,0 \%(4)$ & $14,29 \%(16)$ \\
\hline
\end{tabular}

*Estado com valor estatisticamente significante para variável necessidade de afastamento. 
Tabela 3. Distribuição da freqüência dos participantes segundo a necessidade de afastamento e atividade ocupacional.

\begin{tabular}{|c|c|c|c|c|c|}
\hline \multirow{2}{*}{$\begin{array}{c}\text { Necessidade } \\
\text { de afastamento }\end{array}$} & \multicolumn{5}{|c|}{ Ocupação } \\
\hline & $\begin{array}{c}\text { Costureira, } \\
\text { cabeleireiro e do lar }\end{array}$ & $\begin{array}{l}\text { Construção } \\
\text { civil }\end{array}$ & $\begin{array}{l}\text { Trabalhador } \\
\text { doméstico }\end{array}$ & Lavrador & Segurança \\
\hline Não & $34(68 \%)$ & $5(10,0 \%)$ & $7(14,0 \%)$ & $4(8,00 \%)$ & $0(0,00 \%)$ \\
\hline Sim & $24(38,71 \%) *$ & $10(16,13 \%)$ & $12(19,35 \%)$ & $14(22,58 \%)^{*}$ & $2(3,23 \%)$ \\
\hline
\end{tabular}

*Grupos com valores estatisticamente significativos para variável afastamento.

Tabela 4. Distribuição de afastados que tinham mais de 49 meses de profissão.

\begin{tabular}{llcccc}
\hline \multirow{2}{*}{$\begin{array}{c}\text { Mais de } 49 \\
\text { meses de } \\
\text { profissão }\end{array}$} & $\begin{array}{c}\text { Costureira, } \\
\text { cabeleireiro e do lar }\end{array}$ & $\begin{array}{c}\text { Construção } \\
\text { civil }\end{array}$ & $\begin{array}{c}\text { Trabalhador } \\
\text { doméstico }\end{array}$ & Lavrador & Segurança \\
\cline { 2 - 6 } N ão & $30(71,43 \%)$ & $5(11,90 \%)$ & $4(9,52 \%)$ & $3(7,14 \%)$ & $0(0,00 \%)$ \\
Sim & $16(37,21 \%)$ & $6(13,95 \%)$ & $8(18,60 \%)$ & $13(30,23 \%) *$ & $2(3,23 \%)$ \\
\hline
\end{tabular}

*Grupo com valor estatisticamente significativo para variável mais de 49 meses de profissão emaior tempo de afastamento.

de profissão. Observa-se diferença estatística no maior tempo de profissão, mostrando ter mais indivíduos afastados quando a mesma ocupação for seguida por cinco anos e, ainda, que a atividade de trabal ho rural parece deixar o indivíduo por mais tempo afastado $(p=0,01)$, segundo teste de Pearson.

\section{Discussão}

O afastamento laboral neste estudo acometeu 55\% da amostra estudada, refletindo um problema de saúde com conseqüências socioeconômicas. Segundo a literatura internacional, em 1994, registrou-se que $18 \%$ do total de indenizações por ausência de trabalho foram devidas a problemas nos ombros e pescoço na Suécia e, no estado de Washington, foram 55.315 casos de problemas no ombro de 1981 a 1995. No Brasil, os dados oficiais sobre a prevalência de distúrbios músculo-esqueléticos não apresentam estratificação segundo a região acometida, mas mostram um aumento significativo da concessão de benefícios por lesão por esforço repetitivo no membro su- perior ${ }^{2}$. Além disso, os trabalhadores acreditam que o afastamento éa única possibilidade de evitar as dores e 0 agravamento das lesões ${ }^{19}$.

Em relação à predominância da lesão, os achados desta pesquisa são semelhantes ao da literaturaํ. Todavia, podemos dizer que a freqüência maior de lesão no membro superior direito pode estar relacionada com a dominância e a lesão pode ser resultado de maior sobrecarga me cânica. Além dos aspectos físicos descritos e discutidos, é importante reiterar os comprometimentos econômicos e sociais relacionados às doenças do manguito rotador. Por sua elevada freqüência e grande número de afastamentos, podem ser classificadas como problema de saúde pública20.

O desenvolvimento da afecção do tendão supra-espinal e seus estados podem estar relacionados com a atividade de trabalho, principalmente no que diz respeito à postura, repetição e trabaIhos manuais. Entretanto, sugere-se que, na tendinite, por o quadro doloroso ser maior, as pessoas tendem mais a reclamarem e a se sentirem incapazes de realizar suas tarefas laborai $s^{21-25}$.

Sobre o maior número de afastados no gru- 
po profissional I (costureira, cabeleireiro edo lar) e IV (lavradores), as hipóteses explicativas, mais adequadas para condição, referem-se à redução do aporte sangüíneo durante a flexão de ombro acima de 60 graus e ao impacto constante do manguito rotador sob o arco coracoacromial com carga estática ${ }^{1,14,21,22,23,24,26}$, posturas adotadas no dia-a-dia destes trabalhadores, juntamente com velocidade de movimentos das mãos, o que aumenta a atividade muscular do ombro $0^{8,15,23}$, fatores encontrados no grupo de ramos de serviçose lavradores, diferentes dos indivíduos da construção civil, domésticos e seguranças, que utilizam mais o trabalho de força27.

Em relação à ocupação e afastamento laboral, pôde-se mostrar que, quanto maior o tempo de profissão, maior a probabilidade de estar afastado da função. Alguns autores notaram que 0 tempo de exposição aos fatores de risco é cumulativo e bastante relevante, mostrando que essa relação é mais consistente quando a atividade é maior que cinco anos, concordando com os resultados encontrados em nosso estudo ${ }^{23,28}$. Sobre o tempo de afastamento, o achado maior no grupo delavradores podeser justificado pela idade mais avançada e uso de ferramentas no trabalho ${ }^{28}$, pois estudos relatam esse fator com dor no ombro, já que a atividade repetitiva com as mãos aumenta a atividade muscular do ombro, necessitando de um esforço estático maior para a estabilização do antebraço ${ }^{1,4}$.

A escassez de estudos relacionados, especificamente às variáveis investigadas nesta pesquisa, constitui-se um aspecto limitante para exploração das convergências e divergências com trabaIhos desta natureza e, nesse sentido, optou-se, em alguns casos, pelo levantamento de hipóteses que pudessem sustentar os resultados obtidos. A pesar disso, mesmo nesse âmbito, encontrouse al guma dificul dade, já que as informações sobre as condições biomecânicas foram conseguidas por meio de entrevistas e não pela observação pontual das condições de trabalho.

Apesar das limitações descritas, entende-seque os resultados do presente estudo chamam a atenção para a necessidade de investigar a especificidadedas causas e efeitos relacionados a determinados tipos de atividades e os acometimentos do ombro, especificamentedo tendão supra-espinal, para que métodos de controle dos fatores de risco e de intervenção possam ser mais bem definidos.

A partir dos resultados encontrados, concluiu-se que houve elevado número de casos de afastamentos devido à doença do tendão supraespinal, em maior porcentagem no estado detendinite, nos grupos profissionaisl (costureira, cabeleireiro e do lar) eIV (lavrador), caracterizandose como importante problema epidemiológico.

\section{Colaboradores}

JS Almeida, G Carvalho Filho e NM Lamari participaram integralmente de to das as fases do trabalho, desde o desenho do estudo à redação final. CM Pastre participou do desenho do estudo, da tabulação dos dados e da discussão dos achados. EC Pastre participou do levantamento, seleção de literatura e da discussão dos achados. 
1. Couto HA, Nicoletti SJ, Lech O. Como gerenciar a questão das L.E.R./D.O.R.T. Belo Horizonte: Ergo; 1998.

2. M endonça Jr. HP, Assunção AA. Associação entre distúrbios do ombro e trabalho: breve revisão da literatura. Rev Bras Epidemiol 2005; 8(2):167-176.

3. Helliwell PS, Bennett RM, Littlejohn G, Muirden KD, Wigley RD. Towards epidemiological criteria for soft-tissues disorders of the arm. O ccup $M$ ed 2003; 53:313-19.

4. Van der windt DAM, Thomas E, Pope DP, Winter AF, M acfarlane GJ, Bouter LM, Silman AJ. Occupational risk factors for shoulder pain: a systematic review. Occup Environ M ed 2000; 57:433-442.

5. Ribeiro HP. Lesões por esforço repetitivo: uma doença emblemática. Cad Saúde Pública 1997; 13:85-93.

6. Fuentes $\mathrm{JCH}$. M etodo strain index: aplicado para evaluar trabajos com riesgo de desarrollar trastornos dolorosos de extremidad superior distal. Bol Scient Ass Chil Seg 2003; 5:4-7.

7. Andrade RP, Correa Filho M RC, Queiroz BC. Lesões do manguito rotador. Rev Bras Ortop 2004; 39(11/12): 621-636.

8. Sakata RS, Issey AM. Como diagnosticar e tratar a lesão por esforço repetitivo (LER) doença osteomuscular associada. Rev Bras M ed 2003; 60:77-83.

9. Stenlund B, Goldie I, H agberg M, Hogstedt C. Shoulder tendonitis and its relation to heavy manual work and exposure to vibration. Scand J Work Environ Health 1993; 19:43-9.

10. Carpenter JE, Flanagan CL, Thomopoulus S, Yian EH, Soslowsky LJ. The effects of overuse combined with intrinsec or extrinsec alterations in an animal model of rotator cuff tendonosis. Am J Sports M ed 1998; 26:801-807.

11. Novak CB, Mackinnon SE. Repetitive use and static postures: a source of nerve compression and pain. J Hand Ther 1997;10:151-159.

12. Higgs PE, Edwards DF, Seaton MK, Feely CA, Young $V L$. Age-related differences in measures of upper extremity impairment. J Gerontology 1993; 48(3):175180.

13. Giordano M, Giordano V, Giordano LHB, Giordano JN. Tratamento conservador da síndrome do impacto subacromial: estudo em 21 pacientes. Act Fisiatr 2000; 7:13-19.

14. Filho SBS, Barreto SM. Algumas considerações metodológicas sobre os estudos epidemiológicos das lesões por esforço repetitivo (LER). Cad Saúde Pública 1998;14 (3):555-563.

15. Lech O, Severo A. Ombro e Cotovelo. In: Hebert S, Xavier $\mathrm{R}$, organizadores. Ortopedia e traumatologia- Princípios e prática. 2a ed. Porto Alegre: Artmed; 2003.p.185-236.
16. Luo ZP, Hsu HC, Grabowski JJ, Morrey BF, An KN. $M$ echanical environment associated with rotator cuff tears. J Shoulder Elbow Surg 1998;7:616-620.

17. Volpon JB, Prado MRA. Vascularização do manguito rotador. Rev Bras Ortop 1997;32:699-702.

18. Cordeiro JA. Análise de dependência [tese]. Campinas (SP): U niversidade Estadual de Campinas; 1987.

19. Martins JN, Assunção AA. A dor na doença músculo-esquelética associada ao trabalho. Cad Psicol 2002; 12:61-76.

20. Bertoncello $D$, Almeida A, Alem MER, Walsh IAP, Coury HJCG. Importância da intervenção preventiva da fisioterapia na readequação ergonômica e análise biomecânica de um posto de trabalho. Fisiot M ov 1999; 12:89-96.

21. Leclerc A, Chastang JF, Niedhammer I, Landre M F, Roquelaure $Y$. Incidence of shoulder pain in repetitive work. Occup Environ M ed 2004; 6:39-44.

22. Pope DP, Silman AJ, Cherry NM, Pritchard C, M acfarlane GJ. Association of occupational physical demands and psychosocial working environment with disabling shoulder pain. Am Rheum Dis 2001; 60:852-858.

23. Lin TY, Teixeira MJ, Romano MA, Picarelli $H$, Settini M M, Greve JM D'A. Distúrbios ósteomusculares relacionados ao trabalho. Rev M ed 2001; 80:422-442.

24. Cohen RB, Willians GR. Impingiment syndrome and rotator cuff disease as repetitive motion disorders. Clin Orthop Rel Res 1998; 351:95-101.

25. Mendes R. Patologias do trabalho. São Paulo: Atheneu; 2003.

26. Stenlund B, Lindbeck L, Karlsson D. Significance of house painters' work techniques on shoulder muscle strain during overhead work. Ergonomics 2002; 45:455-468.

27. Ludewig PM, Cook TM. Alterations in shoulder kinematics and associated muscle activity in people with symptoms of shoulder impingement. Phy Ther 2000; 80:276-291.

28. Fredriksson $K$, Alfredsson $L$, Ahlberg $G$, Josephson $M$, Kilbom A, Hjelm EW, Wiktorin C, Vingard E. Work environment and neck and shoulder pain: the influence of exposure time. Results from a population based case-control study. O ccup Environ M ed 2002; 59:182-188.

Artigo apresentado em 29/03/2006

Aprovado em 02/07/2007

Versão final apresentada em 02/08/2007 